\title{
Candida Infection Pathogensis and Host Defensive Mechanism
}

\section{Abdelmoktader A* and Fatma Aboelnaga}

Medical Microbiology and Immunology, Faculty of medicine, Fayoum University, Egypt

*Corresponding author: Abdelrahman Abdelmoktader, Medical Microbiology and Immunology, Faculty of medicine, Fayoum university, Egypt, Tel: 00201006102753 ; Email: aam16@fayoum.edu.eg

\section{Review Article}

Volume 2 Issue 7

Received Date: May 08, 2018

Published Date: May 28, 2018

\section{Abstract}

Candida is a Gram-positive spherical fungi facultative anaerobe, has more than 17 different species and classified into C. albicans and non albicans species. Host response to Candida infection is a complex interplay between innate and adaptive immunity and usually provides a sufficient defense against microbes in healthy individuals but not in immunocompromised ones. Cytokines produced from immune cells also play an important role in epithelial immunity against Candida infection. Candida infection is established by many mechanisms as Adhesins, Invasins, Biofilm, aspartyl proteinases - Haemolysins and Phospholipases.

Keywords: Candida; PRRs; TLRs; GM; CSF; Adhesins; Invasins; Biofilm; Aspartyl Proteinases; Haemolysins; Phospholipases

\section{Introduction}

Candida is a Gram-positive spherical fungi approximately $3 \mu \mathrm{m}$ in diameter. Its cells form budding appearance, since cell division takes place in more than one plane. It is often found as a commensal associated the skin and mucosal surfaces of humans [1].

On an enriched medium, Candida forms variable size white colonies according to species. On sheep blood agar plates, colonies often cause $\beta$-hemolysis due to secretion of hemolysin. Candida is facultative anaerobe capable of generating energy by aerobic respiration, and by fermentation which yields mainly lactic acid [2].

Critically ill or otherwise immunocompromised patients as diabetics, HIV infected individuals and who are under massive antibiotics therapy are more prone to develop both superficial and life-threatening Candida infections [1].

\section{Classification}

Candida can be classified as the followings:

Kingdom: Fungi

Phylum: Ascomycota

Subphylum: Saccharomycotina

Class: Saccharomycetes

Order: Saccharomycetales

Family: Saccharomycetaceae

Genus: Candida and more than 17 different species are known to be etiological agents of human infection. 
Candida species classified into C. albicans and non albicans species [3].

\section{Host Defense Mechanisms against Candida}

Host response to Candida infection is a complex interplay between innate and adaptive immunity and usually provides a sufficient defense against microbes in healthy individuals but not in immunocompromised ones. First, pathogen related receptors (PRRs) are expressed by cells of the innate immune system and specific immunity to recognize pathogen-associated molecular patterns (PAMPs), which are structural components characteristic for microbial pathogens. Recognition of PAMPs leads to microbial uptake and, consecutively, to a modulation of gene to initiate an adaptive and target-oriented immune response [4].

Three main groups of PRRs have been distinguished: toll-like receptors (TLRs), C-type lectin receptors (CLRs) and nucleotide-binding domain leucine-rich repeat containing (NLR) protein family. Each ligand-receptor system activates specific intracellular signaling pathways. Candida cell wall components are mainly recognized extracellularly by Toll-like receptors as in Figure 1 and Ctype lectin receptors on the host cell surface [5].

Mucosalepitheliumisthe first line of defense against Candidaspecies. Epithelial cells express most of the toll like receptors (TLRs), with the exception of TLR5 and TLR7, to recognize invading microorganisms [6]. Recognition of Candida by TLRs leads to different downstream signaling, such as Chemokine/cytokine production and phagocytosis. Once Candida is internalized and phagocytosed, the fungal PAMPs can further activate TLR9 [4].

Polymorphonuclear neutrophils (PMNs) are believed to be the most effective cell type for controlling and clearing Candida infection. In addition to their direct killing of $C$. albicans, it was demonstrated that PMN sare the only cell type in blood which can inhibit C. albicans germ tube formation [7].

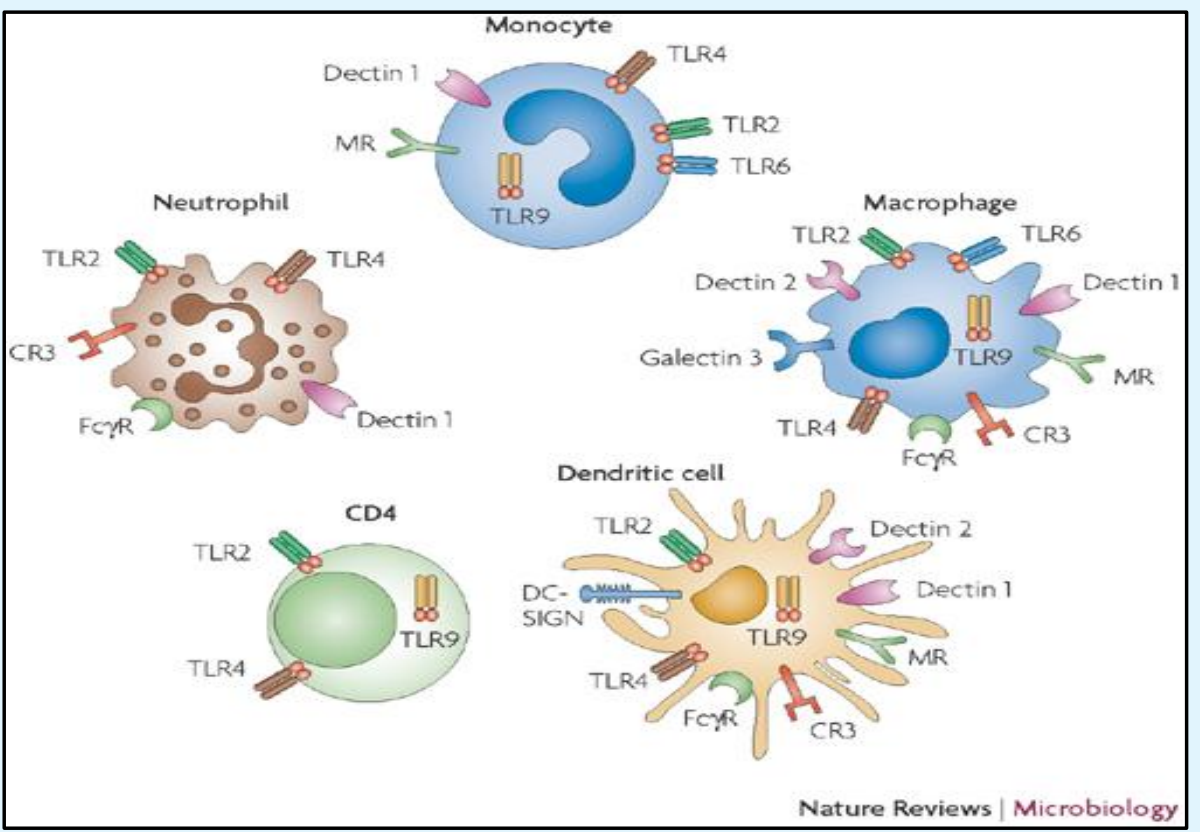

Figure 1: Cell populations and pattern-recognition receptors involved in Candida albicans recognition [5].

Cytokines produced from immune cells also play an important role in epithelial immunity against Candida infection. Several pro-inflammatory cytokines, such as IL6, IL-8, IL-17 and TNF, have been reported to be responsible for the recruitment of PMNs to the site of infection. In addition to pro-inflammatory cytokines, the hematopoietic growth factors granulocyte colonystimulating factor (G-CSF) and granulocyte-macrophage colony-stimulating factor (GM- CSF) are critical for recruitment and activation of PMNs [4]. 
Dendritic cells as professional antigen-presenting cells, DCs reside and patrol in the skin and mucosal surface, and they ingest Candida once tissues are invaded. Candida species are internalized by DCs [8], leading to the processing and presentation of Candida specific antigens via major histocompatibility complex (MHC) class II molecules. DCs discriminate between yeast- and hyphal phase forms of C. albicans and induce $\mathrm{T}$ helper cell differentiation [4].

Ingestion of yeasts primes T helper type 1 cells (Th1), whereas ingestion of hyphae inhibits IL-12 and Th1 differentiation, favoring Th2 differentiation. Thus, DCs bridge the innate and adaptive antifungal responses by recognizing different morphologies of Candida [4].

\section{Candida Pathogenesis}

\section{Adherence to Host Tissue}

Adherence is essential for members of the genus Candida to develop their pathogenic potential, since it triggers the process that leadstocolonization and allows their persistence in the hostand tissue invasion as shown in Figure 2 [9].

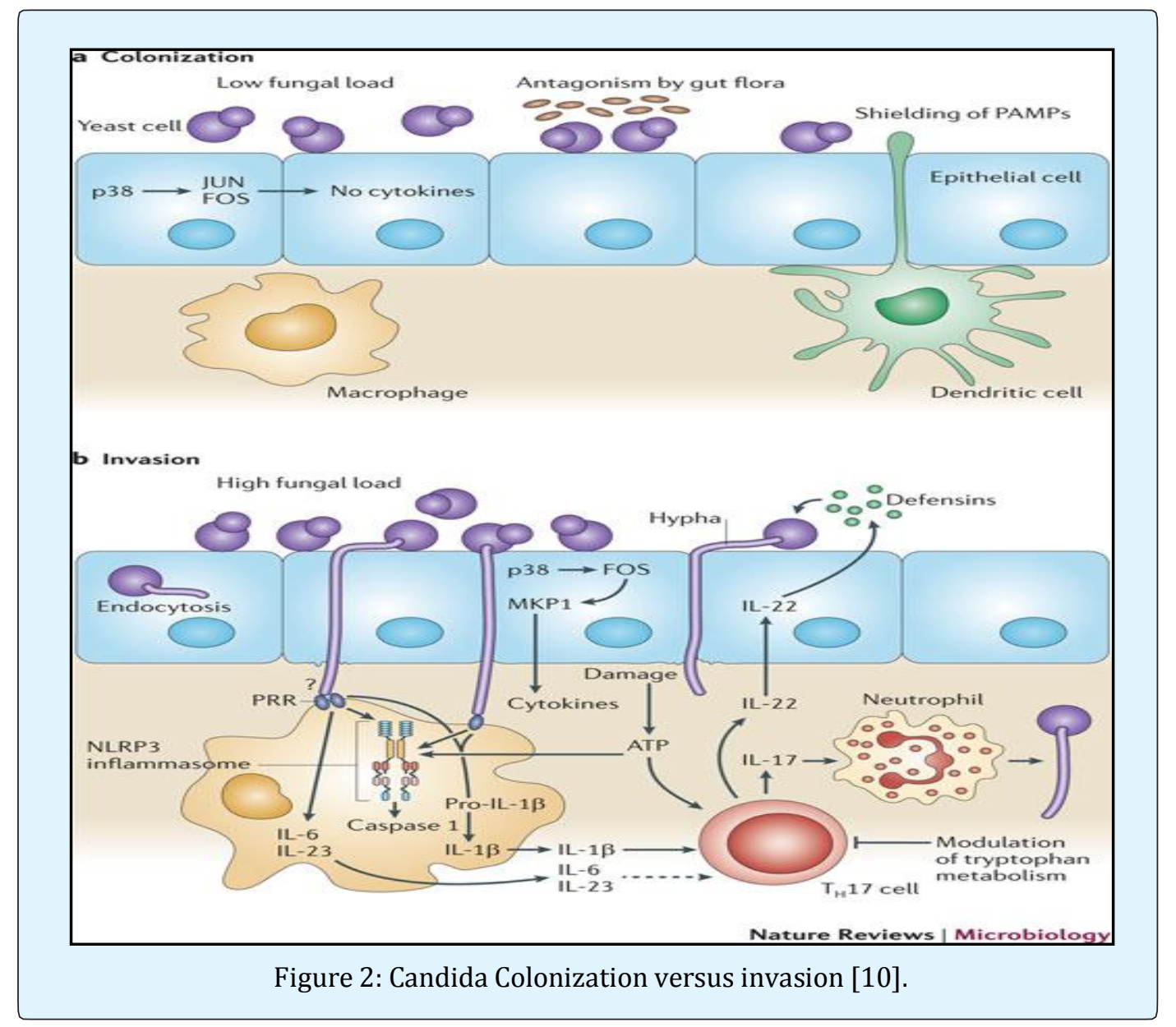

Different intra-species adherence ability has been reported for Candida species [9]. For instance, adherence of $C$. albicans to epithelial cells is greater than that of $C$. parapsilosis [11]. C. tropicalis exhibited higher ability to colonize reconstituted human epithelium (RHE) than did C. parapsilosis and Candida glabrata [12]. Adherence can be mediated through twofamilies of enzymes including adhesines and Invasins.

\section{Adhesins}

C. albicans has a specialized set of proteins (adhesins) which mediate adherence to other $C$. albicans cells, to other microorganisms, to a biotic surface and to host cells. The best studied $C$. albicans adhesins are the agglutininlike sequence (ALs) proteins which form a family consisting of eight members (ALs1-7 and Als9). The ALs genes encode glycosylphosphatidylinositol (GPI)-linked 


\section{Virology \& Immunology Journal}

cell surface glycoproteins. Of the eight ALs proteins, the hypha- associated adhesin ALs3 is especially important for adhesion [13].

The agglutinin-like sequence (ALs3) gene expression is up regulated during infection of oral epithelial cells in vitro and during in vivo vaginal infection [14]. Another important adhesin of C. albicans is Hwp1, which is a hyphae-associated GPI-linked protein. Hwp1 serves as a substrate for mammalian transglutaminases and this reaction may covalently link C.albicans hyphae to host cells [15].

\section{Invasins}

C. albicans is a remarkable pathogen as it can utilize two different mechanisms to invade into host cells: induced endocytosis and active penetration [16].

For induced endocytosis, the fungus expresses specializedproteins on the cell surface (Invasins) that mediate binding to host ligands (such as E-cadherin on epithelial cells and $\mathrm{N}$-cadherin on endothelial cells), thereby triggering engulfment of the fungal cell into the host cell [14].

Indeed, even killed hyphae are taken up, indicating that induced endocytosis is a passive process that does not require the activities of viable fungal cells. Two invasins have been identified so far, namely Als3The agglutininlike sequence (ALs3)(which also functions as an adhesin) and Cell-surface expressed member of the heat shock protein 70 (Hsp70) family (Ssa1) whichpresent on the surface of hypha and plays synergistic role with Als3 [14].

Adhesion between Candida species cells and host cells has been implicated as an early step in biofilm formation [1].

\section{Biofilm}

Biofilms are specific and organized communities of cells under the control of signaling molecules, rather than random accumulations of cells resulting from cell division. C. albicans in a biotic surface biofilms consists of two general types of cells: yeast cells and filamentous cells [17].

In vitro, the basal biofilm layer is composed of yeast cells from which filamentous cells emanate.In Candida the dimorphic transition from the yeast to the hyphal phase is a crucial step in the formation of biofilms, and mutant strains that are defective in the ability to germinate are unable to form dense and homogeneous biofilms [18].
These yeast and filamentous cells are embedded in a dense layer of extracellular matrixthat is self-produced andformed of carbohydrates, proteins, phosphorus and hexosamines. The primary component of biofilm matrix is B-glucan [17].

Environmental conditions such as medium composition, $\mathrm{pH}$ and oxygen as well as the fungal strain and species affect biofilm formation and matrix composition. For example, C. parapsilosis biofilms contain large amounts of carbohydrates, and the protein content is low when compared with biofilms of C. glabrata and $C$. tropicalis [9].

Biofilms can grow on any biotic or a biotic moist surface. The association of organisms in biofilms is a form of protection for their development, encouraging symbiotic relationships and allowing survival in hostile environments [18]. Biofilms may help maintain the role of fungi as pathogenic by evading host immune mechanisms, resisting antifungal treatment and withstanding competitive pressure from other organisms.Biofilm formation by fungi may play an important role in pathogenesis [19]. These biofilms help mixed bacterial and fungal infection found in vaginal infections by Candida species and Gardnerella vaginalis [20].

Recent evidence suggests that the majority of disease produced by C. albicans is associated with biofilm growth [21].

\section{Secreted Hydrolytic Enzymes}

The secretion of hydrolytic enzymes during infection is required as a virulence factor to aid adhesion, invasion, and the destruction of host immune factors, in addition to nutrient acquisition. These enzymes include secreted aspartyl proteinases (SAP), Haemolysins and phospholipases (PL) [22].

\section{Secreted Aspartyl Proteinases}

Secreted aspartyl proteinases (Sap), encoded by a 10members of gene family (SAP1 to SAP10) have been the most extensively studied. The 10 SAP genes that compose this family can be divided into subfamilies based on amino acid sequence homology alignments (SAP1 to SAP3, $S A P 4$ to SAP6, SAP9, and SAP10). These genes exhibit differential expression profiles at different stages and sites of infection [22].

Sap2 is the most highly expressed secreted protease in vitro and is capable of digesting human albumin, hemoglobin, keratin, and secreted immunoglobulin A. 
Sap2 is believed to allow C. albicans to destroy host barriers through the degradation of human proteins, followed by deep penetration into tissues or the bloodstream.In addition, Sap2 digests extracellular proteins into oligopeptides that can be taken up by oligopeptide transporters encoded by the OPT gene family.Therefore, Sap2 may also be critical for cell growth in the human host and may enable the use of host proteins as a nitrogen source. Sap1to- 6 played limited roles in C. albicans virulence [4].

\section{Haemolysins}

Haemolysins are protein produced by micro-organisms to destroy red blood cells for production of iron which is essential for the development ofmicro-organisms, including yeasts, and the ability to obtain this element is essential for the establishment of an infectious process [1].

\section{Phospholipases}

The family of phospholipases consists of four different classes (A, B, C and D). Only the five members of class B (PLB1-5) are extracellular and may contribute to pathogenicity via disruption of host membranes [14] as they catalyze the hydrolysis of phospholipids, which are major components of all cell membranes [23].

\section{Phenotypic and Morphological Switching}

Candida has the remarkable ability to grow in several distinct morphological forms: yeast, hyphae, and pseudohyphae, according to environmental conditions the true hyphae and pseudohyphae (chains of elliptical cells with constrictions at the septa) are often referred to as filamentous forms.The ability to switch rapidly from yeast-to-filamentous growth or vice versa in response to certain environmental factors is considered to be a critical virulence factor for these fungi. In $C$. albicans, each morphologyis believed to confer discrete advantages in the course of infection. The yeast form is important for dissemination through the bloodstream and adheres to endothelial surfaces. The filamentous forms, on the other hand, are more adapted for invasion through the host epithelial tissue and also have a higher resistance to phagocytosis due to their morphology [24].

Candida albicans, strains lacking hyphal formation exhibited lower ability to invade tissue compared with wild-type strains [12].

Indeed, an engulfed C. albicans yeast cell can destroy a macrophage if filamentous growth is triggered after phagocytosis, and filamentous forms have a higher resistance to neutrophil killing .The morphogenesis of $C$. albicans is predominately determined by environmental signaling. The yeast to filamentous transition can be triggered by serum, proline, $\mathrm{N}$-acetylglucosamine, different carbon sources [24].

Candida parapsilosis does not form true hyphae and exists in either a yeast phase or a pseudohyphal form. Differently, C. tropicalis can exist in multiple morphogenetic forms, including yeast phase, pseudohyphae and true hyphae, being considered polymorphic fungi [12].

\section{References}

1. Sardi J, Scorzoni L, Bernardi T, Fusco-Almeida AM, Mendes Giannini MJ (2013) Candida species: current epidemiology, pathogenicity, biofilm formation, natural antifungal products and new therapeutic options. Journal of Medical microbial 62(1): 10-24.

2. Raju SB, Rajappa SS (2011) Isolation and Identification of Candida from the Oral Cavity. ISRN Dentistry 2011: 487921.

3. Lynch DP (1994) Oral candidiasis: History, classification, and clinic presentation. Oral Surg Oral Med Oral Pathol 78(2): 189-193.

4. Cheng SC, Joosten LA, Kullberg BJ, Netea MG (2012) Interplay between Candida albicans and the Mammalian Innate Host Defense. Infect Immun 80(4): 1304-1313.

5. Netea MG, Brown GD, Kullberg BJ, Gow NA (2008) An integrated model of the recognition of Candida albicans by the innate immune system. Nature Reviews Microbiology 6(1): 67-78.

6. Weindl G, Naglik J, Kaesler S, Biedermann T, Hube B, et al. (2007) Human epithelial cells establish direct antifungal defense through TLR4-mediated signaling. J Clin Invest 117: 3664-3672.

7. Fradin C, De Groot P, MacCallum D, Schaller M, Klis F, et al. (2005) Granulocytes govern the transcriptional response, morphology and proliferation of Candida albicansin human blood. Mol Microbiol 56(2): 397-415.

8. Cambi A, Netea MG, Mora-Montes HM, Gow NA, Hato SV, et al. (2008) Dendritic cell interaction with Candida albicans critically depends on N-linked mannan. J Biol Chem 283(29): 20590-20599. 


\section{Virology \& Immunology Journal}

9. Silva S, Negri M, Henriques M, Oliveira R, Williams DW, et al. (2011) Adherence and biofilm formation of non-Candida albicans Candida species. Trends in Microbiology 19(5): 241-247.

10. Gow NA, van de Veerdonk FL, Brown AJ, Netea MG (2011) Candida albicans morphogenesis and host defence: discriminating invasion from colonization. Nature Reviews Microbiology 10(2): 112-122.

11. Lima-Neto RG, Beltrão EI, Oliveira PC, Neves RP (2011) Adherence of Candida albicans and Candida parapsilosis to epithelialcells correlates with fungal cell surface carbohydrates. Mycoses 54(1): 2339.

12. Jayatilake JA, Samaranayake YH, Cheung LK, Samaranayake LP (2006) Quantitative evaluation of tissue invasion by wild type, hyphal and SAP mutants of Candida albicans, and non albicans Candida species in reconstituted human oral epithelium. Journal of Oral Pathology and Medicine 35(8): 481-491.

13. Filler SG, Sheppard DC (2006) Fungal invasion of normally non phagocytic host cells. PloS Pathog 2(12): e129.

14. Mayer FL, Wilson D, Hube B (2013) Candida albicanspathogenicity mechanisms. Virulence 4(2): p119-128.

15. Phan QT, Fratti RA, Prasadarao NV, Edwards JE Jr, Filler SG (2005) N-cadherin mediates endocytosis of Candida albicans by endothelial cells. J Biol Chem 280(11): 10455-10461.

16. Finkel JS, Mitchell AP (2011) Genetic control of Candida albicans biofilm development. Nat Rev Microbiol 9(2): 109-118.
17. Jabra-Rizk MA, Shirtliff M, James C, Meiller T (2006) Effect of farnesol on Candida dubliniensis biofilm formation and fluconazole resistance. FEMS Yeast Res 6(7): 1063-1073.

18. Davey ME, O'toole GA (2000) Microbial biofilms: from ecology to molecular genetics. Microbiol Mol Biol Rev 64(4): 847-867.

19. Martinez LR, Fries BC (2010) Fungal biofilms: relevance in the setting of human disease. Curr Fungal Infect Rep 4(4): 266-275.

20. Harriott MM, Noverr MC (2011) Importance of Candida-bacterial polymicrobial biofilms in disease. Trends Microbiol 19(11): 557-563.

21. Ganguly S, Mitchell AP (2011) Mucosal biofilms of Candida albicans. Curr Opin Microbiol 14(4): 380385.

22. Correia A, Lermann U, Teixeria L, Cerca F, Botelho S, et al. (2010) Role of Saps in murins disseminated candidasis infection. Immun 78.

23. Samaranayake YH, Dassanayake RS, Jayatilake JA, Cheung BP, Yau JY, et al. (2005) Phospholipase B enzyme expression is not associated with othervirulence attributes in Candida albicans isolates from patients with human immunodeficiency virus infection. Journal of Medical Microbiology 54: 583593.

24. Han TL, Cannon RD, Villas-Bôas SG (2011) The metabolic basis of Candida albicans morphogenesis and quorum sensing. Fungal Genetics and Biology 48: 747-763. 